GENETICS

\section{Backing Boveri}

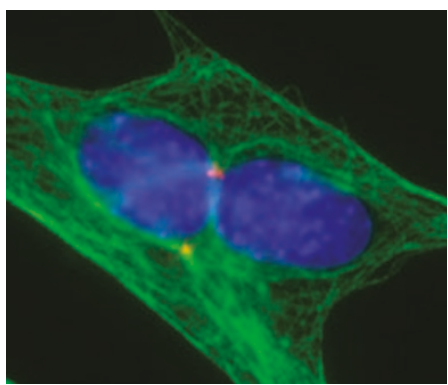

The idea that mitotic defects that result in tetraploidy promote oncogenesis was proposed over 100 years ago by Theodor Boveri, and although numerous subsequent studies have supported this concept, proving it has been a challenge. In a study published in Nature, David Pellman and colleagues developed a system to directly compare p53-null diploid and tetraploid cells (see image), and have shown that tetraploid cells have greater tumorigenic potential.

To generate genetically matched tetraploid and diploid cells, Pellman's group started out with p53-null mouse mammary epithelial cells (MMECs). Loss of p53 has been previously associated with tetraploidy in various tumour cell types. Cytokinesis was blocked in primary MMECs that were isolated from $\operatorname{Tr} p 53^{+/+}$or $\operatorname{Tr} p 53^{-/-}$mice by treatment with the cytoskeletal disrupting agent dihydrocytochalasin B. Diploid (40 chromosomes) and tetraploid (80 chromosomes) cells were then isolated by fluorescence-activated cell sorting.

The authors observed that the diploid and tetraploid cultures of p53-null cells proliferated at similar rates in culture, but the Trp53 $53^{+/+}$cells did not form tetraploids that could be propagated. With further analysis of the p53-null cells, Pellman's group also found that although wholechromosome aneuploidy arose in both the diploid and tetraploid populations, a higher percentage of the tetraploid-derived cells developed aneuploidy. Many of these cells also had gross chromosomal rearrangements.
What is the impact of tetraploidy on transformation? Neither the diploid nor the tetraploid p53-null cells were initially able to undergo anchorage-independent growth. However, when both cell types were treated with the mutagen 7,12-dimethylbenz[a] anthracene (DMBA), followed by exposure to the tumour promoter 12-Otetradecanoylphorbol-13-acetate (TPA), the tetraploid cells alone developed the ability to grow in soft agar. These cells went on to form tumours within two weeks when injected into nude mice. In fact, the p53-null tetraploid cells were able to form tumours in vivo without being exposed to DMBA or TPA - out of 39 animals injected with untreated tetraploid cells, 10 developed tumours at the site of injection. By contrast, no tumours formed at sites where p53-null diploid cells were injected.

Genetic analysis of the tetraploid-derived tumour cells revealed extra centrosomes, numerical and structural chromosome aberrations, as well as abundant non-reciprocal translocations and dicentric chromosomes. Interestingly, each of the 9 tumours examined had amplifications in a region of chromosome 9 that contains a cluster of 10 matrix metalloproteinase genes, which are commonly overexpressed in human breast tumours.

The authors conclude that a transient block in cytokinesis, leading to the formation of genetically unstable tetraploid cells, promotes tumorigenesis in MMECs. However, further experiments are required to determine how often tetraploidy occurs in normal cells and what causes it (see further reading).

Kristine Novak

\section{(2) References and links}

ORIGINAL RESEARCH PAPER Fujiwara, T. et al.

Cytokinesis failure generating tetraploids promotes tumorigenesis in p53-null cells. Nature $\mathbf{4 3 7}$, 1043-1047 (2005)

FURTHER READING Shi, Q. \& King, R. W. Chromosome nondisjunction yields tetraploid rather than aneuploid cells in human cell lines. Nature 437, 1038-1042 (2005)

\section{TRIAL WATCH}

\section{Tumour-draining lymph nodes}

The presence of lymph node metastases is a strong negative predictor of disease-free survival (DFS) and overall survival in patients with breast cancer. Sentinel node biopsy sample analysis accurately predicts axillary node metastasis and therefore identifies women who do not need surgery to remove axillary nodes. Analysis of the immune cell populations in tumourdraining lymph nodes of breast cancer patients now shows that immune profiling could be a useful additional prognostic factor.

Holbrook Kohrt et al. examined 47 sentinel and 104 axillary lymph nodes from 77 breast cancer patients with sentinel lymph node involvement and 5-year follow up, to see if changes in the $\mathrm{CD}^{+}$and $\mathrm{CD}^{+} \mathrm{T}$ cell or $\mathrm{CD} \mathrm{a}^{+}$dendritic cell populations predicted nodal metastases or DFS. They found that the numbers of immune cells were decreased in sentinel and tumour-draining axillary nodes compared with control nodes in breast cancer patients. The authors used 29 patients as a training set and showed that axillary nodes with greater than or equal to $7.0 \%$

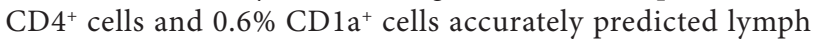
node metastasis. It was confirmed in a test set of 48 patients that this immune profile could successfully stratify the patients into favourable and unfavourable groups. Axillary node, but not sentinel node, $\mathrm{CD} 4^{+} \mathrm{T}$ cell and dendritic cell numbers correlated well with DFS. This stratification performed better than traditional clinicopathological methods of prognosis, indicating that changes in the immune profile in tumour-draining lymph nodes might precede tumour invasion.

ORIGINAL PAPER Kohrt, H. E. et al. Profile of immune cells in axillary lymph nodes predicts disease-free survival in breast cancer. PLoS Med. 2, 904-919 (2005)

\section{TGF $\beta$ polymorphisms}

Epidemiological studies have shown that the ${ }^{\star} 6 \mathrm{~A}$ polymorphism in the type I transforming growth factor $\beta$ receptor gene (TGF $\beta R 1)$ might be a tumour susceptibility allele. So, Boris Pasche et al. examined tumour and germline tissue from cancer patients to find out whether this allele was somatically acquired by primary tumours and metastases.

The authors examined tissue from 531 patients with colorectal, head and neck, or breast cancer, and found that $2.5 \%$ of colorectal tumours, $1.8 \%$ of head and neck tumours and no breast tumours had somatically acquired TGF $\beta R 1^{\star} 6 A$. In addition, $29.5 \%$ of liver metastases from colorectal cancer had somatically acquired $T G F \beta R 1^{\star} 6 A$. The polymorphism was not associated with loss of heterozygosity, microsatellite instability or a mutator phenotype.

The $6 \mathrm{~A}^{*}$ polymorphism causes a three-amino-acid deletion in the signalling sequence of mature TGF $\beta R 1$, so Pasche et al. investigated whether this deletion affected the translocation of the receptor to the cell membrane or TGF $\beta R 1$ signalling, which might explain the acquisition of this allele in tumours and metastases. They found that TGF $\beta R 1$ and TGF $\beta R 1^{*} 6$ A produced identical mature receptors in MCF-7 breast cancer cells and DLD- 1 colorectal cancer cells, but that TGF $\beta \mathrm{R} 1^{\star} 6 \mathrm{~A}$ might switch TGF $\beta$ growth inhibitory signals into growth stimulatory signals.

The authors suggest that TGF $\beta \mathrm{R} 11^{\star} 6 \mathrm{~A}$ might represent a potential therapeutic target in cancer.

ORIGINAL PAPER Pasche, B. et al. Somatic acquisition and signalling of TGF $\beta R 1^{\star} 6 A$ in cancer. JAMA 294, 1634-1646 (2005) 